\title{
Skeletal System
}

National Cancer Institute

\section{Source}

National Cancer Institute. Skeletal System. NCI Thesaurus. Code C12788.

The collection of bones and tissues, including ligaments and cartilage, that compose the rigid internal framework of the human body, and which serves a multitude of functions, including structural support, movement, protection, production of blood cells, storage of ions, and endocrine regulation. 